\title{
On the Fundamental Plane of the Galactic globular cluster system
}

\author{
M. Pasquato ${ }^{1}$ and G. Bertin ${ }^{2}$ \\ 1 Dipartimento di Fisica, Università di Pisa, Largo Bruno Pontecorvo 3, 56127 Pisa, Italy \\ 2 Dipartimento di Fisica, Università degli Studi di Milano, via Celoria 16, 20133 Milano, Italy
}

Received 27 January 2008 / Accepted 9 May 2008

\begin{abstract}
Context. The globular clusters of our Galaxy have been found to lie close to a plane in the $\log R_{\mathrm{e}}, \log \sigma, S B_{\mathrm{e}}$ space, on the continuation of the Fundamental Plane that is known to characterize the global properties of early-type galaxies. There is no apparent reason why such physically different self-gravitating systems should follow the same scaling law.

Aims. We reexamine the issue by focusing on a sample of 48 globular clusters selected with homogeneity criteria for the photometric data available from the literature.

Methods. We perform a model-independent analysis of surface brightness profiles and distance moduli, estimating error bars and studying selection effects with robust non-parametric statistical tests.

Results. We determine the values of the coefficients that define the Fundamental Plane and their error bars and show that the scatter from the Fundamental Plane relation is likely to be intrinsic, i.e. not due to measurement errors only. Curiously, we find that in the standard Fundamental Plane coordinates the set of points for our sample occupies a rather slim, axisymmetric, cylindrical region of parameter space, which suggests that the relevant scaling relation might be around a line, rather than a plane, confirming results noted earlier. This is likely to be the origin of the difficulties in the fit by a plane, often mentioned in previous investigations. In addition, such a Fundamental Line relation would imply a pure photometric scaling law relating luminosity to the effective radius which might be tested on wider samples and on extra-galactic globular cluster systems. As to the residuals from the Fundamental Plane relation, we find a correlation of the deviations from the plane with the central slope of the surface brightness profile. No other statistically significant correlations are identified. Finally, given the constraint imposed by the virial theorem, we study the distribution of the values of the quantity $K_{V} /(M / L)$ (virial coefficient divided by the relevant mass-to-light ratio); the distribution of the logarithms, reconstructed through kernel density estimation methods, shows evidence for bimodality, which suggests that the galactic globular cluster system may be composed of at least two dynamically different populations. Yet, these populations do not appear to reflect the standard dichotomy between disk and halo clusters.
\end{abstract}

Key words. Galaxy: globular clusters: general - Galaxy: structure

\section{Introduction}

It is well known (Dressler et al. 1987; Djorgovski \& Davis 1987) that early-type galaxies occupy a region in the vicinity of the plane $\log R_{\mathrm{e}}=\alpha \log \sigma_{0}+\beta S B_{\mathrm{e}}+\gamma$ in the three-dimensional parameter space defined in terms of the projected effective radius ${ }^{1} R_{\mathrm{e}}$, of the central velocity dispersion $\sigma_{0}$, and of the mean surface brightness $S B_{\mathrm{e}}$. This Fundamental Plane (FP) exhibits a tilt, i.e. the measured values of the coefficients that characterize the plane differ significantly from the naive expectations based on the application of the virial theorem. The properties of the FP partly reflect the characteristics of the stellar populations in these systems and partly some systematic deviations from strict structural homology (e.g., see Bertin et al. 2002).

It is often stated that the universality of the FP extends well outside the domain of early-type galaxies and applies to many other systems, such as globular clusters (hereafter GCs; see Djorgovski 1995; McLaughlin 2000; Barmby et al. 2007), "cluster spheroids" (Zaritsky et al. 2006), open clusters (Bonatto \& Bica 2005), X-ray emitting gas in elliptical galaxies (Diehl \& Statler 2005), clusters of galaxies (see Lanzoni et al. 2004, and references therein), and even more exotic objects, such as

\footnotetext{
1 Some authors employ $r_{\mathrm{e}}, R_{\mathrm{h}}$, or $r_{\mathrm{h}}$ to refer to the projected effective (half-light) radius. In our notation $r_{\mathrm{e}}$ refers to the projected effective angular radius, while $R_{\mathrm{e}}$ to the projected effective linear radius.
}

supermassive black holes (e.g., Hopkins et al. 2007) and quasars (e.g., Hamilton et al. 2006). Comparisons of the FP relation for different systems are often present in the literature: for example, see Burstein et al. (1997) and Martini \& Ho (2004), who also address the problem of the mass gap between high-mass GCs and low-mass galaxies and related classification issues in the socalled $k$-space introduced by Bender et al. (1992).

In particular, for galactic GCs it has been found by Djorgovski (1995) that an FP relationship based on core parameters (core radii and central surface brightness) holds and that it is "consistent with globular cluster cores being virialized systems with a universal and constant $M / L$ ratio", while for quantities referred to the effective radius $\left(R_{\mathrm{e}}\right.$ and $\left.S B_{\mathrm{e}}\right)$ the FP does not emerge as sharply, possibly because of error correlations (see additional remarks in Sect. 3 below). In reality, since GCs are non-homologous stellar systems with rather homogeneous stellar populations, it would be surprising if they happened to follow a continuation of the FP of early-type galaxies, which appear to be associated with very different structural properties, in terms of homology, stellar populations, and dark matter content.

In this paper we thus reconsider the problems of the existence and of the nature of the FP for the GCs of the Milky Way Galaxy. Although galactic GCs are extremely well studied objects, for a proper statistical investigation of their structural properties the use of the entire set of data available from 
the literature suffers from several limitations since the GCs are distributed over the entire sky, the relevant photometric and spectroscopic data that have been collected come from different telescopes and different instruments. In addition, GCs are located at different distances from the Sun and, in the sky, at very different ranges of galactic longitude and latitude, thus suffering from different amounts of reddening. Thus different groups of GCs suffer from different uncertainties in distance determination, which has a major impact on the determination of the intrinsic properties that enter the FP. All this makes the entire set of data highly inhomogeneous and thus not well suited to a proper statistical investigation.

As a preliminary step for a satisfactory statistical investigation, we identify a sample of galactic GCs that has optimal characteristics, being sufficiently large while being associated with sufficiently homogeneous data of their photometric properties. Sample selection is a delicate issue, so this problem is thoroughly discussed in the following.

The paper is organized as follows. In Sect. 2 we describe the criteria that led us to identify our optimal sample of GCs and summarize its properties. In Sect. 3 we study the possible existence of the FP in the relevant parameter space and show that, in the standard FP coordinates, our sample occupies a rather slim cylindrical region, which suggests that the scaling relation might be around a line, rather than a plane, a result already noted in previous investigations (Bellazzini 1998, see also Burstein et al. 1997). In Sect. 4 we discuss two unexpected features, i.e. a residual correlation with a parameter characterizing the central slope of the brightness profile and a bimodality in the distribution of the effective virial coefficients $K_{V} /(M / L)$, reconstructed by means of kernel density estimation methods. In Sect. 5 we discuss the selection effects in our sample in comparison with those present in previous investigations (in particular, in Djorgovski 1995). In Sect. 6 we summarize the results obtained and present discussion and conclusions.

\section{The adopted sample}

Since our primary objective is to test whether the galactic GCs follow a continuation of the FP of early-type galaxies, we refer to the standard global photometric parameters, i.e. to the effective radius and to the related mean surface brightness $\left(R_{\mathrm{e}}\right.$ and $\left.S B_{\mathrm{e}}\right)$, which are commonly used in the study of the FP of early-type galaxies (for a complete definition, see Bertin et al. 2002) and to ignore other options (such as the use of core parameters, $R_{0}$ and $S B_{0}$; see Djorgovski 1995). The criteria for the identification of our sample, extracted from the data set available in the literature, are largely dictated by the requirement of accurate distance determination.

In the following four subsections, we address separately the main ingredients required for an accurate determination of the parameters entering in the FP relation, i.e. distance moduli, reddening, surface brightness profiles, and line-of-sight velocity dispersions.

\subsection{Distance moduli}

The two most recent papers that contain an extensive set of galactic globular clusters for which distance moduli have been determined with a uniform method (using the Zero Age Horizontal Branch (ZAHB) as a standard candle) and have been assigned individual error bars are Ferraro et al. (1999), who studied and uniformly reduced colour-magnitude diagrams from several sources, for a set of $61 \mathrm{GCs}$ and that by
Recio-Blanco et al. (2005), based on an extensive photometric data set from HST for 72 GCs (Piotto et al. 2002). The samples overlap partially. Different procedures are used in the two papers for data reduction, particularly with respect to the determination of the ZAHB level. Recio-Blanco et al. (2005) compare their distance moduli whith those of Ferraro et al. (1999) at the intersection of the two samples, and find them on average larger than those of Ferraro et al. (1999) with a mean offset of $0.09 \mathrm{mag}$. Moreover, an rms scatter of $0.17 \mathrm{mag}$ is found for the differences, with some values being as discrepant as 0.4 mag. Therefore, in this paper, we decided to consider the properties of a combined sample, which contains 48 GCs (see Sect. 2.5), but also to check the behaviour of two sub-samples studied separately (see Appendix).

In their Table 2, Ferraro et al. (1999) list ZAHB magnitude levels, with the related photometric error bars, and two sets of true distance moduli $\left(|m-M|_{0}\right)$, based on two different assumptions on the enhanced abundance of $\alpha$-elements (the so-called $[\alpha / \mathrm{Fe}]$ enhancement) of GC stars with respect to solar abundances. Column 7 of that table lists the distance moduli obtained by assuming solar abundances (i.e., with no $[\alpha / \mathrm{Fe}]$ enhancement) and $[\mathrm{Fe} / \mathrm{H}]$ from Carretta \& Gratton (1997), while column 8 lists the distance moduli obtained by adopting $[\alpha / \mathrm{Fe}]$ values from the literature (Salaris \& Cassisi 1996; Carney 1996) and by mimicking $\alpha$-enhanced isochrones by standard scaled solar models of suitable metallicity, as proposed by Salaris et al. (1993). The authors state that distance moduli "are affected by many uncertainties (namely, the evaluation of the ZAHB level, the zero point and dependence on metallicity of the ZAHB level, reddening, etc.)" so that "the global uncertainty affecting the distance moduli listed in Table 2 cannot be less than 0.2 mag".

If we linearly interpolate the distance moduli as a function of $[\alpha / \mathrm{Fe}]$ in the interval between 0 and the value of $[\alpha / \mathrm{Fe}]$ adopted for the construction of Col. 8, we can take into account the uncertainty on $[\alpha / \mathrm{Fe}]$ by adding to the distance moduli error the product between the relative error in $[\alpha / \mathrm{Fe}]$ and the difference between the distance moduli listed in Table 8 and those in Table 7. This appears to be reasonable. In view of the sparseness and heterogeneity of $[\alpha / \mathrm{Fe}]$ data, as noted also by Ferraro et al. (1999), we estimate the relative error on this quantity to be approximately $50 \%$. With that, we can take into account this factor in the uncertainty on distance moduli. Keeping in mind the level of 0.2 mag suggested by Ferraro et al. (1999), we linearly transform the error bars obtained above, so that the first quartile of the error bar size distribution was rescaled to $0.2 \mathrm{mag}$ and the third quartile to $0.3 \mathrm{mag}$. This procedure is both reasonable and statistically robust, because it is not affected by outliers in the error bar size distribution and turns out to be compatible with the scatter and systematic differences that arise when comparing distances obtained from different standard candles, such as the Main Sequence or the White Dwarf Cooling Sequence (for a distance measurement of NGC 104 based on the White Dwarf Cooling Sequence, see Zoccali et al. 1999), or when comparing the distance moduli of Ferraro et al. (1999) with those of Recio-Blanco et al. (2005).

The distance moduli of Recio-Blanco et al. (2005) are probably of higher quality than those of Ferraro et al. (1999) because they are based on uniform HST photometry, at least as far as random error bars are concerned. For the intersection of the two samples our adopted uncertainties are therefore obtained by summing in quadrature to the error estimates by Recio-Blanco et al. (2005) the semi-difference between the distance moduli obtained by Recio-Blanco et al. (2005) and those by Ferraro et al. (1999). For the remaining clusters with distance moduli 
only in Recio-Blanco et al. (2005), we assume a constant difference equal to the mean difference quoted above, and use it for an analogous quadrature sum to obtain the adopted error bars.

Our conservative procedure may tend to overestimate the error bars on distance moduli. Still we will see that the formal reduced $\chi^{2}$ for the FP fit are larger than unity.

\subsection{Reddening}

Recio-Blanco et al. (2005) provide independent measurements of the reddening affecting the GCs in their sample, which we averaged with those reported in the McMaster Catalogue (Harris 1996). For the GCs not considered by Recio-Blanco et al. (2005), we adopted the values reported in the latter Catalogue. On the values of reddening, we assigned error bars as suggested by Harris: "the typical uncertainty in the reddening for any cluster is on the order of 10 percent, i.e. $\delta[E(B-V)]=0.1 E(B-V)$ ". Note that the apparent distance moduli determined by Recio-Blanco et al. (2005) refer to the F555W HST band; to de-redden them, we thus applied the relevant reddening/extinction relationship, obtained from Table 12 of Holtzman et al. (1995) through a linear fit of the form $A_{F 555 W}=k E(B-V)$. This differs from the usual $A_{V}=3.1 E(B-V)$ in the value of the proportionality coefficient $k$.

\subsection{Surface brightness profiles: apparent magnitudes and effective radii}

To calculate apparent effective radii $r_{\mathrm{e}}$ and apparent $V$-band magnitudes (or equivalently, the values of mean $V$-band surface brightness within the effective radius, $S B_{\mathrm{e}}$ ) we started from the circularized surface brightness profiles reported by Trager et al. (1995). A more recent compilation (McLaughlin \& van der Marel 2005) of GC surface brightness profiles and structural parameters is available, comprising both Milky Way and extragalactic GCs, but, for the galactic GCs, it is based on fitting various models to the same set of data by Trager et al. (1995). Our model independent approach thus prompted us to proceed independently, basing our analysis directly on the Trager et al. (1995) profiles. These profiles are based on data from different sources and do not quote explicit error estimates. On the other hand, the authors provide some quality rating of the data, based on the reliability of the data-set from which they were extracted. These weights, $w$, ranging from 0.0 (totally unreliable data-set) to 1.0 (high quality data-set) were admittedly "assigned by eye" (Trager, private communication). Relative error bars might be introduced as $\sigma \propto 1 / \sqrt{w}$, but this would still be somewhat arbitrary. Eventually, to set the magnitude of the error bar for each surface brightness data-point, we resorted to the (halved) residuals to the Chebyshev polynomial fits to the profiles provided by Trager et al. (1995), to avoid relying on a subjective assessment of data quality. This choice has its own limitations, because such residuals depend on the particular form of the fitting function, are partly correlated, and may include as an error term some spurious deviations (e.g., deviations associated with the presence of a bright star).

The physical parameters that we wish to derive require integration of the profiles on the radial coordinate. Since the points are not evenly spaced in radius and are rather noisy, we smooth and interpolate the data by cubic splines prior to integration (see also Trager et al. 1995). We then integrate using different methods (e.g., trapezoidal and Simpson's rule) and checked that the results are stable. The integration error was obtained by

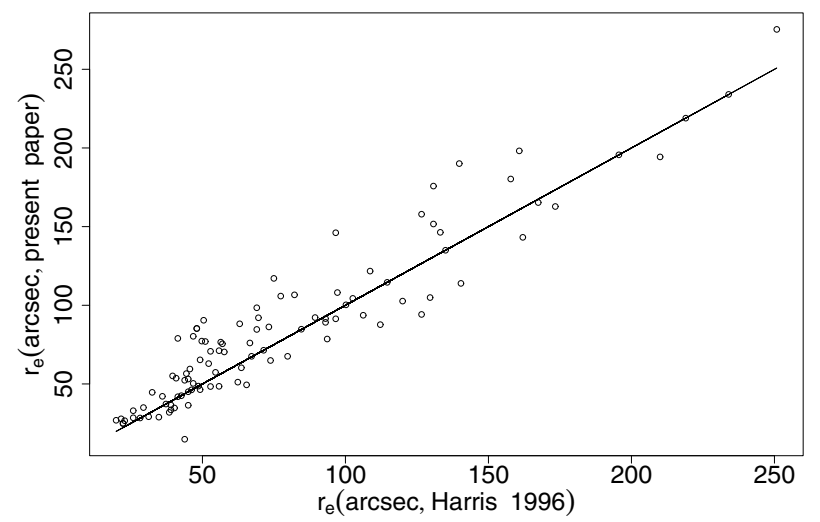

Fig. 1. Comparison of apparent effective radii reconstructed in this paper with those reported in the McMaster Catalogue (Harris 1996). A 1:1 line has been drawn to guide the eye.

comparing results derived by changing the number of interpolating points and checked to be at least one order of magnitude smaller than other sources of error.

The surface brightness profiles were extrapolated beyond the last available data-point, which is set by the merging of the profile into the background sky brightness and so differs from one GC to another. To extrapolate, we used a linear relation between surface brightness and angular radius. We tested a number of alternatives for an optimal extrapolation and noted that the result did not change the derived total magnitude by more than $0.05 \mathrm{mag}$.

The apparent effective radii were then determined in a straightforward manner by building a curve of growth through integration of the profile and finding the radius at which the integrated luminosity equals half of the total luminosity. Obviously, since uncertainty on the total magnitude propagates to the effective radius, this factor has the potential of introducing an anticorrelation between uncertainties on the two quantities (see also Sect. 3.1 of Treu et al. 1999). The importance of this error term, which in the error budget for the effective radius competes with the error deriving from the uncertainty on the parameters of the interpolation curve, depends strongly on the steepness of the growth curve in the vicinity of the effective radius.

Figure 1 shows a comparison of the values of effective radii obtained in this paper with those recorded in the McMaster Catalogue (Harris 1996). Following Trager et al. (1995), we replaced points characterized by excessive discrepancies with values from the literature (in our case from Harris 1996). This was performed by replacing only those data that we found to be outliers in the distribution of differences. For this purpose, we defined as an outlier a point that lies outside the interval $\left[Q_{1}-\left(Q_{3}-Q_{1}\right), Q_{3}+\left(Q_{3}-Q_{1}\right)\right]$, with $Q_{1}$ and $Q_{3}$ being the first and third quartile of the data distribution; this is slightly more conservative than the more common definition based on $\left[Q_{1}-1.5\left(Q_{3}-Q_{1}\right), Q_{3}+1.5\left(Q_{3}-Q_{1}\right)\right]$.

Our angular radii are slightly larger than those listed in the McMaster Catalogue, showing a median difference of 0.04 in the logarithm. The standard deviation of the differences is somewhat larger, at 0.10 , but still compatible with our estimated error bars on effective radii. Similarly, our magnitudes exceed those of Harris (1996) by 0.07 mag in the median (i.e., we find clusters to be systematically dimmer), and the differences show a standard deviation of $0.21 \mathrm{mag}$. This scatter is slightly higher than that expected based on error estimates for our sample alone.

Unfortunately, the surface brightness profiles presented by Trager et al. (1995) do not allow us to carry out the analysis 
in different colors. Therefore, we will be unable to address the possible impact of mass segregation on the FP scatter.

\subsection{Velocity dispersions}

We took the line-of-sight velocity dispersions from Pryor \& Meylan (1993), which are complete with uncertainties estimated by the authors. The term central velocity dispersion (and the corresponding subscript 0 ) would be misleading in this context; so, while we have used it when referring to the FP of elliptical galaxies, we will not use it in the following, because the data set contains some dispersions that are not strictly referred to the center of the cluster. We did not try to rescale these measurements to a common aperture size, because this would be, to some extent, model dependent (e.g., if we made use of King (1966) models; see also McLaughlin \& van der Marel (2005) and Table 13 therein for observed central velocity dispersions corrected using different models). In the adopted data set two kinds of velocity dispersion measurements are mixed, from integrated light spectroscopy and from the kinematics of individual stars. In a total of 56 GCs, 31 have integrated light measurements and 35 have stellar kinematic measurements, while only 10 have both.

Integrated light measurements make use of a small (several arcseconds) spectroscopic slit centered on the GC. In contrast, samples of stars used for stellar kinematics often span a much wider angle (up to several arcminutes). In addition, integrated light spectroscopy cannot discriminate between the cluster and background or foreground sources, nor can it exclude fast moving binary stars which may exaggerate the observed velocity dispersion. Moreover, the light collected by the spectroscopic slit may be dominated by a small number of bright giants, therefore contributing to poor statistics. On the other hand, stellar samples used for kinematics are usually selected carefully for membership and tested against binaries through repeated observations, but may be rather small and unevenly distributed in the cluster; they also are often biased towards highly luminous stellar types (mainly red giants). All these factors contribute to producing a systematic difference between these two kinds of measurements, which we have tested on the subsample of clusters having both kinds of measurement and found to be significant. In Sect. 4.2, devoted to the analysis of the distribution of virial coefficients, we will restrict our study to the relatively homogeneous subsample of GCs for which the velocity dispersion is given by integrated light measurements, because the virial coefficient is proportional to the square of the velocity dispersion and sample homogeneity is crucial. For the rest of the paper, we repeated our calculations on the restricted subsample and checked that the results are in general agreement with those obtained on the larger, unrestricted sample.

\subsection{The sample}

The McMaster Catalogue of Milky Way Globular Cluster Parameters (Harris 1996) lists 151 globular clusters. The sample we selected is much smaller, comprising only 48 globular clusters. Moreover, as explained below, data inhomogeneity has forced us to separately consider also two smaller subsamples for which homogeneous measurements of distance moduli are available.

Since we are aiming at a model-independent approach, we have carefully excluded from our sources those studies that provide exclusively model-dependent quantities (in particular, surface brightness profiles from King model fits). Since we wish to determine error bars on the FP coefficients, we have restricted our attention to data with objective estimates of uncertainties. Finally, we have given priority to homogeneous data-sets (i.e. data from the same authors, taken with the same instruments, and reduced in a similar fashion).

In conclusion, our sample has been selected as the intersection of three data-sets: one with well-determined distance moduli obtained by merging Ferraro et al. (1999) and Recio-Blanco et al. (2005), one with well-determined line-of-sight velocity dispersions (Pryor \& Meylan 1993), and one with surface brightness profiles (Trager et al. 1995) from which we could recompute apparent magnitudes and effective radii. The properties of our sample of $48 \mathrm{GCs}$ are summarized in Table A.1. In the Appendix we describe the behaviour of two smaller sub-samples that are obtained by considering either Ferraro et al. (1999) or Recio-Blanco et al. (2005) separately.

\section{The "Fundamental Plane"}

After extracting distance moduli $|m-M|_{0}$ and reddening values $E(B-V)$ from our sources and reconstructing the apparent effective radii $r_{\mathrm{e}}$ and apparent integrated magnitudes $m$, we choose the two photometric variables that enter the FP from the three definitions (unit-dependent constants, which play a role only in determining the zero point, are set by measuring $R_{\mathrm{e}}$ in kpc, $r_{\mathrm{e}}$ in $\operatorname{arcsec}$, and $S B_{\mathrm{e}}=41.40-2.5 \log \left(L / 2 \pi R_{\mathrm{e}}^{2}\right)$ in mag/arcsec ${ }^{2}$ where $L$ is expressed in units of the solar $V$-band luminosity ${ }^{2}$ ):

$\log R_{\mathrm{e}}=\frac{1}{5}|m-M|_{0}+\log r_{\mathrm{e}}-7.31$

$S B_{\mathrm{e}}=m-3.1 E(B-V)+5 \log r_{\mathrm{e}}+1.99$,

$M=m-|m-M|_{0}-3.1 E(B-V)$.

We assume that errors on distance moduli and reddening values do not correlate with errors on apparent effective radii and integrated apparent magnitudes. On the other hand, as noted earlier, some degree of anti-correlation is present between apparent magnitudes and effective radii (see Treu et al. 1999, for a discussion of correlations between analogous parameters of the FP of early type galaxies). From Eq. (2) above, we see that $S B_{\mathrm{e}}$ is the only photometric coordinate of the parameter space that does not depend on distance moduli (although it depends on the reddening). Therefore, as long as errors on distance moduli dominate the error budget, to optimally deal with the problem of error correlations, it is appropriate to choose from the three photometric parameters listed above any of the other two photometric quantities together with $S B_{\mathrm{e}}$. Even if the contribution of the error on $\log r_{\mathrm{e}}$ to the error on $S B_{\mathrm{e}}$ is comparable to that of the error on distance moduli, it is partly cancelled by the anti-correlated error on the apparent magnitude $m$.

These arguments confirm that the standard use of the two photometric variables $\log R_{\mathrm{e}}$ and $S B_{\mathrm{e}}$ is justified.

For an ordinary least square linear regression in the threedimensional parameter space defined by $\log R_{\mathrm{e}}, S B_{\mathrm{e}}$, and $\log \sigma$, we have to choose which coordinate to take as dependent variable, the other two being regarded as independent variables. In the literature the most common choice as the dependent variable is $S B_{\mathrm{e}}$, which is known to provide "a better and more stable fit" with respect to other options (Djorgovski 1995). Different choices give different results, and it is not clear which one is the

\footnotetext{
2 Our source, Trager et al. (1995), gives surface brightnesses in the $V$ band, although it is actually based on data taken in different passabands.
} 
best. This is related to the rather narrow distribution of the cluster data points on the FP viewed face on (i.e. to the datapoints lying more around a line than a plane, see discussion in the following sections), a point that we have tested by a set of simple simulations. If the data lie approximately on a line in parameter space, then any plane passing through that line is a good fit to the data. The best fit is different when different variables are chosen as dependent variables because the sum of squared residuals along that coordinate is minimized; in this way, the fitting algorithm chooses the best-fit plane almost at random among the infinitely many planes that pass through the line, by fitting the scatter around such a line. $S B_{\mathrm{e}}$ :

We find the following fit by the Fundamental Plane based on

$S B_{\mathrm{e}}=(3.28 \pm 0.57) \log R_{\mathrm{e}}-(3.59 \pm 0.39) \log \sigma+(27.95 \pm 2.94),(4)$

In turn, fitting through $\log R_{\mathrm{e}}$ we obtain:

$\log R_{\mathrm{e}}=(0.13 \pm 0.02) S B_{\mathrm{e}}+(0.30 \pm 0.13) \log \sigma-(4.94 \pm 0.50),(5)$

and, finally, through $\log \sigma$ :

$\log \sigma=-(0.19 \pm 0.01) S B_{\mathrm{e}}+(0.42 \pm 0.12) \log R_{\mathrm{e}}$

$$
+(5.07 \pm 0.46) \text {. }
$$

In all these fits, we weighted the data-points by the inverse squared uncertainty on the relevant dependent variable, neglecting other uncertainties. The constant term assumes that angular effective radii are measured in arcseconds, velocity dispersions in kilometers per second, distance moduli and integrated apparent luminosities are measured in magnitudes, and surface brightness is expressed in magnitudes per square arcsecond (see also the beginning of this section).

The relatively large uncertainties on the fit coefficients are not the result of overestimating error bars on data points. In fact, the reduced $\chi^{2}$ for the FP fits is rather high: 9.56, 4.38 and 18.88, respectively. These values derive either from underestimating observational errors (which is probably not the case, given the conservative approach taken in this paper) or, more likely, from the presence of intrinsic scatter in the FP relationship. We will address this issue further in the next section, by discussing the correlations between the residuals to the FP and other GC observational parameters.

It is customary (see Djorgovski 1995) to re-express the FP in terms of $\log R_{\mathrm{e}}$, even if we prefer, as argued above, to carry out the fit through $S B_{\mathrm{e}}$. This involves an algebraic manipulation of the coefficients obtained from the $S B_{\mathrm{e}}$ based fit. Therefore, to obtain error bars for the derived coefficients it is natural to apply standard error propagation techniques, thus obtaining from Eq. (4):

$\log R_{\mathrm{e}}=(1.09 \pm 0.31) \log \sigma+(0.30 \pm 0.05) S B_{\mathrm{e}}-(8.52 \pm 2.35),(7)$

which can be easily compared to the results of other studies or to theoretical predictions. We should recall that error bars computed through standard error propagation techniques are not necessarily accurate when uncertainties are large, because such techniques are linear. Figure 2 shows an edge-on view of the FP. The scatter in $\log R_{\mathrm{e}}$ is approximately 0.15 .

Equation (4) can be compared to previous results, e.g. Eq. (6) in Djorgovski (1995), which we report here in our notation:

$S B_{\mathrm{e}}=(2.9 \pm 0.1) \log R_{\mathrm{e}}-(4.1 \pm 0.2) \log \sigma+(19.8 \pm 0.1)$.

The coefficients we obtain by fitting through $S B_{\mathrm{e}}$ are compatible, within two sigma, with the results by Djorgovski (1995), while

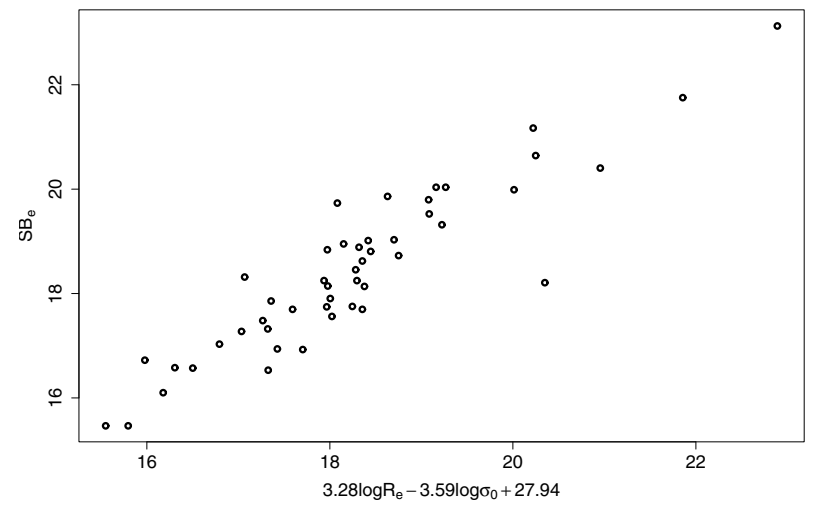

Fig. 2. Edge-on view of the FP for the galactic GCs.

the zero point is not, due to different conventions in measurement units. The results in Eqs. (4)-(7) are interesting because they demonstrate the difficulty in fitting by a plane, as discussed in Sect. 3.1

The FP coefficients found in Eq. (7) by fitting through $S B_{\mathrm{e}}$ and inverting for $\log R_{\mathrm{e}}$ are consistent with those of the FP for early-type galaxies, also in the zero-point constant, namely (Jørgensen et al. 1996):

$\log R_{\mathrm{e}}=1.24 \log \sigma_{0}+0.33 S B_{\mathrm{e}}-8.895$,

with a reported scatter of 0.08 in $\log R_{\mathrm{e}}$, but the error bars are marginally compatible even with the zero-tilt plane:

$\log R_{\mathrm{e}}=2.0 \log \sigma+0.40 S B_{\mathrm{e}}+\gamma$.

This result shows that more accurate data are required in order to draw convincing physical conclusions.

\subsection{A Fundamental Line?}

If on the FP viewed face-on the data-points are located mainly within a thin strip (with width comparable to the scatter around the FP) it may be difficult to discriminate between a plane distribution and a line distribution. In principle we could argue that, by suitable rescaling of the coordinates, any plane distribution may be stretched into a line distribution and vice versa, although we should check how the coordinate transformation changes the relevant error bars. Rather than following this apparent paradox as a general problem of statistics, here we adopt the view that the standard choice of coordinates used in discussions of the FP has its own physical justification and we check whether, in the standard FP coordinates, the set of points occupies a slim cylindrical region of parameter space or not. We thus calculate the eigenvalues and eigenvectors of the tensor of inertia of the unweighted data-points in the $\left(S B_{\mathrm{e}}-\left\langle S B_{\mathrm{e}}\right\rangle, \log R_{\mathrm{e}}-\left\langle\log R_{\mathrm{e}}\right\rangle, \log \sigma-\langle\log \sigma\rangle\right)$ coordinates (i.e. in the "center of mass" frame) and obtain for the eigenvalues $I_{1}, I_{2}$, and $I_{3}$ :

$I_{1}=1.99$

$I_{2}=120.68$

$I_{3}=121.33$

and for the respective eigenvectors (in the aforementioned coordinates):

$v_{1}=(0.98,0.09,-0.15)$ 
$v_{2}=(0.05,0.67,0.74)$

$v_{3}=(0.17,-0.73,0.66)$.

The eigenvector corresponding to the smallest eigenvalue sets the direction of the "Fundamental Line". This procedure is equivalent to unweighted least square fitting in terms of a Fundamental Line (i.e. finding the straight line that minimizes the sum of distances to the points squared) but also gives a measure of the degree of axisymmetry of the distribution of datapoints around such a Fundamental Line, in terms of the difference between the other two eigenvalues $I_{2}$ and $I_{3}$ :

$R \equiv \frac{2\left|I_{2}-I_{3}\right|}{\left(I_{2}+I_{3}\right)}=0.005$,

which is remarkably small.

Indeed, it was already noted (Burstein et al. 1997; Bellazzini 1998) that the galactic GCs lie close to a straight line in the $\log R_{\mathrm{e}}, S B_{\mathrm{e}}, \log \sigma$ parameter space. But, to the best of our knowledge, the present paper is the first to identify and quantify the degree of axisymmetry around such a line, a finding that demands an adequate theoretical explanation. Since Bellazzini (1998) suggests that "at earlier times, globular clusters populated a line in the three-dimensional S-space, i.e. their original dynamical structure was fully determined by a single physical parameter", we also looked for correlations between residuals to the Fundamental Line and GC relative age (taken from De Angeli et al. 2005), finding only a very small, but positive, correlation coefficient of 0.05 , which further decreases to 0.03 if more robust non-parametric correlation estimators (such as the Spearman estimator (e.g., see Wasserman 2006)) are used. However, this apparently negative result is not conclusive, because our sample contains mainly metal-poor clusters (see Sect. 5), and De Angeli et al. (2005) clearly state that "the age dispersion for the metalpoor clusters is $0.6 \mathrm{Gyr}(\mathrm{rms})$, consistent with a null age dispersion". To clarify this point a larger sample is needed.

\subsection{A pure photometric scaling law?}

A Fundamental Line relation of the type identified above would imply the existence of a pure photometric scaling law approximately of the form

$\log R_{\mathrm{e}} \propto \frac{1}{10} S B_{\mathrm{e}}$

i.e.

$L \propto R_{\mathrm{e}}^{-2}$.

This result is unexpected because the trend suggested by Eq. (19) would be qualitatively opposite to that of the Fish (1964) law. In addition, on a sample of 143 GCs, Djorgovski \& Meylan (1994) conclude that no significant correlation exists between integrated absolute magnitude and the logarithm of the effective radius. From the relevant plot in their Fig. 6, we note that an underlying trend might be masked by the presence of two or three outlier points; furthermore, in their Table 1 Djorgovski \& Meylan (1994) report a positive correlation (with $r=0.819$ ) between $\log R_{\mathrm{e}}$ and $S B_{\mathrm{e}}\left(\left\langle\mu_{V}\right\rangle_{\mathrm{h}}\right.$ in their notation), which can be recast into a scaling law of the form of Eq. (18), recovering the correct sign for the coefficient.

On the other hand, the correlation that we find in the photometric plane is in qualitative agreement with the general trend noted for elliptical galaxies by Kormendy (1977); see also
Hamabe \& Kormendy (1987); Capaccioli et al. (1992). We have checked that, for the sample of 88 GCs obtained by intersecting the two samples of Ferraro et al. (1999) and Recio-Blanco et al. (2005) with that of Trager et al. (1995), the correlation that we find in the photometric plane indeed has a consistent slope but a different zero point with respect to the relation $\mu_{e V}=$ $2.94 \log R_{\mathrm{e}}+19.48$ reported by Hamabe \& Kormendy. To be sure, the $\mu_{\mathrm{e} V}$ variable is very sensitive to the quality of the surface brightness profiles; therefore, a direct comparison with the least squares regression of Kormendy (1977) in the $\mu_{\mathrm{e} V}$ variable cannot be performed easily.

The value of the exponent in Eq. (19) is very sensitive to the adopted fitting procedure. The projected Fundamental Line is not equal to the straight line that minimizes the sum of the squared residuals along one coordinate in the plane $\left(\log R_{\mathrm{e}}, S B_{\mathrm{e}}\right)$, i.e. it does not coincide with the line one would obtain with simple linear least squares fitting. To recover the same relation as predicted by the projected Fundamental Line, one should perform an impartial regression in the $\left(\log R_{\mathrm{e}}, S B_{\mathrm{e}}\right)$ plane, i.e. one should minimize the sum of squared distances to the line, not just the residuals in one coordinate. Moreover, it should be noted that the least squares method implicitly used for fitting the Fundamental Line is non-robust, i.e. its results may be excessively influenced by outliers; this is obviously true also for the Fundamental Line projection and the derived scaling relation.

The scatter around the Fundamental Line has the potential to significantly blur the univariate correlation obtained by projecting the Fundamental Line on the photometric $\left(\log R_{\mathrm{e}}, S B_{\mathrm{e}}\right)$ plane. Therefore, we anticipate that either a narrowing of the sample to a smaller set of GCs or the identification of a variable $\eta$ capable of reducing that scatter ( $\eta$ could be the central slope of the surface brightness profile, as in the case of the FP, or age as suggested by Bellazzini 1998) might lead to a much improved univariate relation between $\log R_{\mathrm{e}}$ and $S B_{\mathrm{e}}$. Possibly, a new bivariate correlation could be sought for in the $\left(\log R_{\mathrm{e}}, S B_{\mathrm{e}}, \eta\right)$ space.

A clear interest in a scaling law of the form of Eq. (19) lies in the fact that its study does not require the acquisition of kinematical information. Therefore, we should be able to test it on wider samples and possibly on globular cluster systems in external galaxies. This will be addressed in a follow-up paper. The exclusion of the $\log \sigma$ coordinate would also allow us to consider samples that are more homogeneous from the statistical point of view. Finally, the existence of a pure photometric scaling relation suggests that the physical basis for the empirical scaling should be sought more in the context of stellar populations than within the virial theorem and dynamical arguments.

\section{Two unexpected features}

\subsection{Correlation of residuals with the central slope of the surface brightness profile}

The scatter around the FP, as quantified by the value of the reduced $\chi^{2}$ significantly greater than unity might be interpreted as an indication of the role of nonlinearities or of a fourth parameter.

We looked for residual correlations with the coordinates defining the space in which the FP lies, namely $\log R_{\mathrm{e}}, S B_{\mathrm{e}}$, and $\log \sigma$. By looking at the relevant scatter plot, the residuals show no recognizable pattern with either $\log R_{\mathrm{e}}$, or $S B_{\mathrm{e}}$, or $\log \sigma$; from a qualitative point of view, this suggests that nonlinear corrections to the FP, if present, are unobservable, at least with current data quality. Quantitatively, the null hypothesis that the coefficients of nonlinear terms in the form $\left(\log R_{\mathrm{e}}\right)^{2}$, 


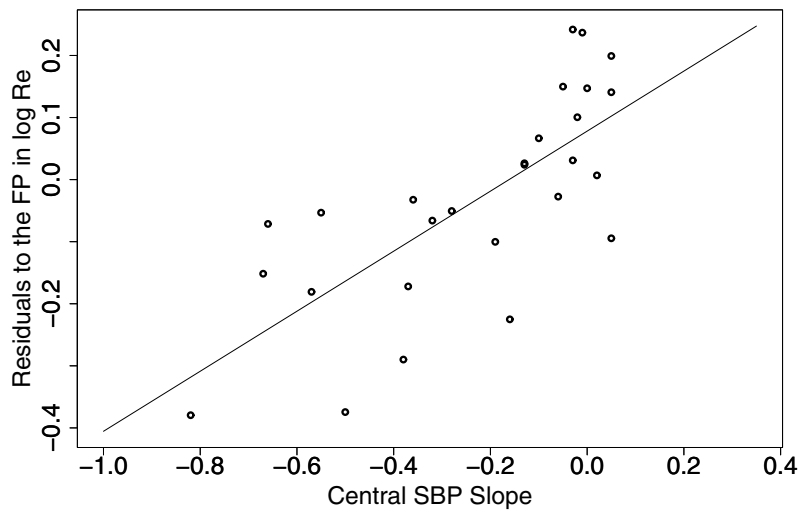

Fig. 3. Correlation of the FP residuals with slope of the central surface brightness profile as defined and measured by Noyola \& Gebhardt (2006).

$\log R_{\mathrm{e}} \log \sigma, \ldots$, are equal to 0 cannot be refuted even at a $20 \%$ significance level.

We consider correlations with other quantities, mainly of dynamical interest. In particular, we examined the King model concentration parameter, central slope of the surface brightness profiles (as defined and measured by Noyola \& Gebhardt 2006), position in the Galaxy (i.e. galactocentric distance and vertical distance from the galactic disk), age, relaxation time referred to the effective radius, metallicity $[\mathrm{Fe} / \mathrm{H}]$, and colour $B-V$. Our investigation is not complete, because other quantities, in particular colour-magnitude-diagram related quantities, were not addressed.

Consistent with indications from previous studies (e.g., see Djorgovski \& Meylan 1994) which excluded correlations between GC properties, even in the case of FP residuals no significant correlations are found, with just one interesting exception, a statistically significant correlation of the FP residuals with the central slope of the surface brightness profile. In view of the results obtained by McLaughlin (2000) (see also Barmby et al. 2007), a trend with galactocentric position would be expected; the fact that the present analysis is unable to detect it could be due to the selection effect in galactocentric distance characterizing our sample. Figure 3 shows a plot of FP residuals against the central slopes taken from Noyola \& Gebhardt (2006). The trend of smaller effective radii with increasing slopes is evident from the scatter plot; the value of the related correlation coefficient is 0.73 . The presence of such a correlation reinforces the view that the origin of the FP scatter is indeed intrinsic. This result is particularly interesting because it relates central to global properties of these stellar systems, much like the $M_{B H^{-}} \sigma$ relationship (Ferrarese \& Merritt 2000; Gebhardt et al. 2000) does for bulges and elliptical galaxies. Indeed, an analogous argument has been proposed by Faber et al. (1997) in their study of the core regions of elliptical galaxies and spiral bulges; in particular, Faber et al. (1997) state that "Cores follow a fundamental plane that parallels the global fundamental plane for hot galaxies but is $30 \%$ thicker. Some of this extra thickness may be due to the effect of massive black holes (BHs) on central velocity dispersions". We note that in some GCs the gravitational sphere of influence of an intermediate mass black hole (such has been argued by Noyola et al. (2008) to be present in Omega Cen) could have an angular size comparable to the slit size used for integrated light measurements of the velocity dispersion. This effect might be at the origin of the observed correlation; fitting cuspy GC cores with double-power law profiles (see Byun et al. 1996) or with simple

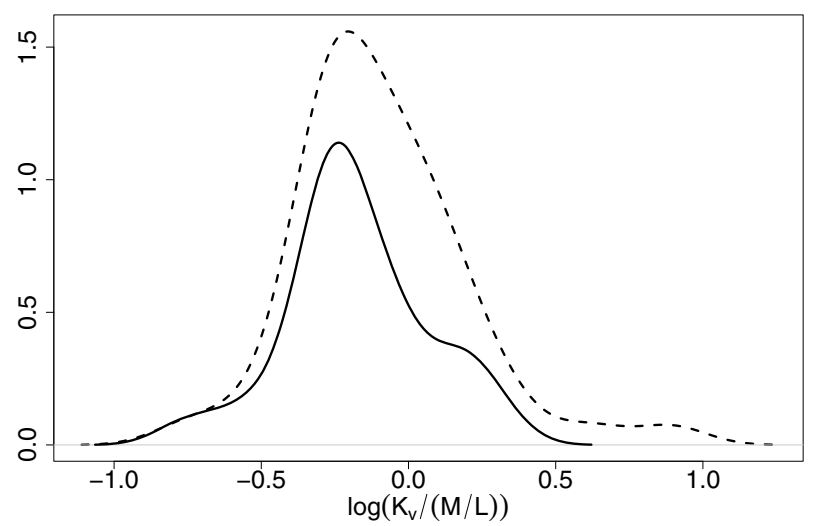

Fig. 4. Probability density distribution of $\log K_{V} /(M / L)$ for clusters with velocity dispersions measured in integrated light (solid line) and for the entire sample (dashed line), reconstructed by application of the non-parametric method of kernel density estimation. Units have been chosen in such a way that $K_{V} /(M / L)=1$ for NGC 104 .

dynamical models (Cipollina \& Bertin 1994) might shed further light on this question.

We have also checked whether the scatter in the FP can be related to the presence, in our sample, of objects that are suspected to be non-genuine galactic GCs, such as members of GC streams (Lynden-Bell \& Lynden-Bell 1995), which are probable remnants of GC systems tidally stripped from a smaller galaxy accreted by the Milky Way. We have compared the distribution of residuals to the FP for those GCs that are part of such streams (according to Gao et al. 2007) with that of the rest of our sample through a Kolmogorov-Smirnov test and found no evidence of significant differences. Therefore, we argue that GC streams are not responsible for the excessive FP scatter, or, in other words, that, in this context, stream GCs are dynamically similar to native GCs.

\subsection{Distribution of effective virial coefficients}

We have reconstructed the distribution of $K_{V} /(M / L) \equiv$ $G L /\left(R_{\mathrm{e}} \sigma^{2}\right)$ (virial coefficient divided by the mass-to-light ratio; for a thorough discussion, see Bertin et al. 2002) for our sample, making use of kernel density estimation techniques (see Wasserman 2006). In many respects, these non-parametric techniques are an extension of histograms, best suited to reveal structure that histograms may hide. The $K_{V} /(M / L)$ distribution looks bimodal, even if we choose a slightly larger bandwidth than optimal (1.2 times) so as to err on the side of oversmoothing (undersmoothing could easily produce fictitious bi- or multi-modal distributions). The indication of bimodality is stronger if the analysis is restricted to the 28 systems for which the line-of-sight velocity dispersion is obtained from integrated light measurements, while for the entire sample of $48 \mathrm{GCs}$ it almost disappears. Figure 4 illustrates the reconstructed probability density distribution of $\log K_{V} /(M / L)$ for the restricted and the unrestricted case, with the restricted case showing the superposition of a narrow peak around a negative value onto a broader distribution with a positive mean. Therefore the inhomogeneity of velocity dispersion measurements is not responsible for the bimodality, which appears more clearly when the sample is restricted to integrated light velocity dispersion measurements only. This result is also suggestive of an interpretation in terms of the presence of intermediate mass black holes, which would naturally affect the value of the central velocity 
dispersion (best diagnosed by the integrated light measurements) in some of the globular clusters, but, as for the feature noted in the previous subsection, a thorough investigation on a larger sample would be required in order to make a fully convincing case. Even though the mass-to-light ratio of a stellar population may be heavily influenced by its metallicity, we feel confident in excluding a metallicity-dependent effect as responsible for the bimodality of the $\log K_{V} /(M / L)$ because no correlation is observed between effective virial coefficient and metallicity; using $[\mathrm{Fe} / \mathrm{H}]$ data from the McMaster Catalogue, we find a correlation coefficient of 0.04 between these two quantities, which is statistically compatible with zero.

In addition, this result makes a point that argues against the zero tilt FP scenario by showing that the $\log K_{V} /(M / L)$ values have a non-trivial distribution, i.e. inconsistent with a sharply peaked distribution broadened into a bell-shaped curve by observational errors.

\section{Selection effects}

As noted earlier, the McMaster Catalogue of Milky Way Globular Cluster Parameters (Harris 1996) lists 151 globular clusters, while the sample considered in the present paper is much smaller, comprising 48 GCs. By comparison, the sample used by Djorgovski (1995) contains 55 globular clusters, those for which a measurement of line-of-sight velocity dispersions is available in Pryor \& Meylan (1993). Under these conditions a thorough study of selection effects is required.

First, we defined a list of relevant parameters, extracted from those tabulated in the 2003 version of the McMaster Catalogue, namely the distance of the GC from the Galactic Center $R_{\mathrm{gc}}$, the vertical distance to the Galactic Plane $Z$, the distance from the Sun $d_{\odot}$, the absolute integrated magnitude $M_{V}$, the integrated colour $B-V$ (uncorrected for reddening), the metallicity as measured by the $[\mathrm{Fe} / \mathrm{H}]$ parameter, the King model concentration parameter $c$, the projected effective radius $R_{\mathrm{e}}$, the logarithm of the relaxation time referred to the effective radius $\log t_{\mathrm{e}}$, and the central surface brightness $\mu_{V}$. We then compared the distribution of these parameters of our sample with that of the complementary sample, that is, of the sample containing all of the GCs in the McMaster catalogue except those of our sample.

In assessing the importance of selection effects, we used the quantities taken from the McMaster Catalogue; we did not try to recompute any of them anew for consistency reasons, because we would otherwise treat our sample (for which quality data are available) in a different way to the rest of the clusters.

The comparison has been performed with a non-parametric Kolmogorov-Smirnov test. The test gives an estimate of the probability of the sample and of its complementary set being drawn from the same distribution. If this probability is extremely low, say less than $10^{-3}$, we can quite confidently assert that a selection effect is present, i.e. that the sample we considered is not statistically representative of the whole population of galactic GCs. Being non-parametric, this test does not assume a given functional dependence for the distribution function of the data, which is unknown in principle; this is an important requirement in this context, because the distribution functions of the various parameters we considered are often far from Gaussian and may be highly skewed or have long tails.

We performed a similar test for the wider sample of 55 GCs with velocity dispersions measured by Pryor \& Meylan (1993). Djorgovski (1995) assumes no relevant selection effects on that sample, at variance with our analysis, that shows that some selection effects are indeed present at a significant level.
Table 1. Comparison of the $48 \mathrm{GC}$ sample of this paper with the complementary sample within the McMaster Catalogue, from which the data listed in this table are taken.

\begin{tabular}{llllll}
\hline \hline $\mathrm{ID}$ & $p_{K \& S^{a}}{ }^{a}$ & $\mu_{\mathrm{s}}{ }^{b}$ & $\mu_{\mathrm{c}}{ }^{c}$ & $\sigma_{\mathrm{s}}{ }^{d}$ & $\sigma_{\mathrm{c}}{ }^{e}$ \\
\hline$R_{\mathrm{gc}}(\mathrm{kpc})$ & 0.2 & 10.05 & 12.96 & 13.62 & 22.02 \\
$Z(\mathrm{kpc})$ & 0.1 & 1.94 & 0.53 & 8.13 & 18.09 \\
$d_{\odot}(\mathrm{kpc})$ & 0.2 & 11.72 & 16.80 & 12.41 & 20.60 \\
$M_{V}$ & 0.0002 & -7.78 & -6.69 & 1.07 & 1.58 \\
$B-V$ & 0.004 & 0.90 & 1.18 & 0.27 & 0.48 \\
{$[\mathrm{Fe} / \mathrm{H}]$} & 0.1 & -1.45 & -1.22 & 0.49 & 0.58 \\
$c$ & 0.0006 & 1.82 & 1.44 & 0.54 & 0.54 \\
$R_{\mathrm{e}}(\mathrm{pc})$ & 0.04 & 3.63 & 4.94 & 3.22 & 4.68 \\
$\log t_{\mathrm{e}}(\mathrm{yr})$ & 0.2 & 9.09 & 9.04 & 0.39 & 0.54 \\
$\mu_{V}$ & $8 \times 10^{-7}$ & 16.99 & 19.82 & 2.01 & 2.66 \\
\hline
\end{tabular}

${ }^{a} p$-value for the null hypothesis of the sample and its complementary sample coming from the same distribution.

${ }^{b}$ Mean value of the relevant parameter in the sample.

${ }^{c}$ Mean value of the relevant parameter in the complementary sample.

${ }^{d}$ RMS of the relevant parameter in the sample.

${ }^{e} \mathrm{RMS}$ of the relevant parameter in the complementary sample.

Table 2. Comparison of the 55 GC sample of Djorgovski (1995) with the complementary sample within the McMaster Catalogue. See Table 1 for column heading explanations.

\begin{tabular}{llllll}
\hline \hline $\mathrm{ID}$ & $p_{K \& S}$ & $\mu_{\mathrm{s}}$ & $\mu_{\mathrm{c}}$ & $\sigma_{\mathrm{s}}$ & $\sigma_{\mathrm{c}}$ \\
\hline$R_{\mathrm{gc}}(\mathrm{kpc})$ & 0.1 & 9.70 & 13.38 & 12.90 & 22.72 \\
$Z(\mathrm{kpc})$ & 0.5 & 1.54 & 0.66 & 7.71 & 18.73 \\
$d_{\odot}(\mathrm{kpc})$ & 0.1 & 11.33 & 17.39 & 11.94 & 21.13 \\
$M_{V}$ & $2 \times 10^{-5}$ & -7.85 & -6.57 & 1.15 & 1.52 \\
$B-V$ & 0.002 & 0.90 & 1.21 & 0.26 & 0.49 \\
{$[\mathrm{Fe} / \mathrm{H}]$} & 0.02 & -1.48 & -1.19 & 0.46 & 0.59 \\
$c$ & 0.0009 & 1.79 & 1.43 & 0.52 & 0.56 \\
$R_{\mathrm{e}}(\mathrm{pc})$ & 0.04 & 3.62 & 5.05 & 3.04 & 4.84 \\
$\log t_{\mathrm{e}}(\mathrm{yr})$ & 0.2 & 9.11 & 9.02 & 0.40 & 0.54 \\
$\mu_{V}$ & $2 \times 10^{-8}$ & 16.96 & 20.06 & 1.94 & 2.58 \\
\hline
\end{tabular}

Our statistical tests are summarized in Table 1 (our sample against the rest of galactic GCs) and Table 2 (the Pryor \& Meylan (1993) sample against the rest of galactic GCs), which also list the mean and standard deviation of the chosen sample and of its complementary sample. Kolmogorov-Smirnov p-values are approximated to one significant digit, while all other numbers are truncated to the second significant digit.

The results of our statistical analysis can be summarized as follows. Selection effects are definitely present, although they do not necessarily alter the conclusions of the present paper. Both samples (Djorgovski 1995 and ours) are biased in favor of clusters relatively close to the Sun; both are biased towards intrinsically more luminous and centrally brighter clusters. These selection effects, although expected, must be kept in mind when addressing issues such as whether the FP properties change with Galactocentric distance.

On the other hand, it is somewhat surprising to find that both samples are biased in the direction of bluer and more centrally concentrated clusters (even though this latter feature may partly result from the fact that the McMaster Catalogue assigns a concentration parameter of 2.5 to GCs recognized to be in the Post Core Collapse phase of their evolution). Concentration parameters show a trend with GC integrated luminosities (see Djorgovski \& Meylan 1994), so the bias towards concentrated clusters might be a byproduct of the selection effect in 
magnitude. Our sample has a somewhat lower mean metallicity than average; this selection effect becomes even stronger if only the subsample with distance moduli from Ferraro et al. (1999) is used (see Appendix).

The clusters in the Djorgovski (1995) sample are slightly smaller than average, in terms of $R_{\mathrm{e}}$, while for both samples there seems to be no bias in the relaxation time referred to $R_{\mathrm{e}}$. Therefore, we feel confident in excluding major dynamical differences between the two samples (Djorgovski 1995 and ours) and the overall population of galactic GCs, which makes it reasonable to extend our conclusions to the whole system of galactic GCs.

Selection effects might be present in more than one variable taken together, even when they do not appear when considering only one variable at a time, as we did. That is, if $x$ and $y$ are two variables, the distribution function of our sample could be different from that of the rest of the GCs on the $(x, y)$ plane, while being indistinguishable when projected onto the $x$ or the $y$ axes. This issue is beyond the scope of the present paper.

\section{Summary, discussion, and conclusions}

\subsection{Results}

Based on the identification of an optimal sample of 48 globular clusters selected based on homogeneity criteria for the photometric data available in the literature, the most important results of the present study are the following:

- We have determined the coefficients of the FP for the galactic globular cluster system, with error bars (see Eq. (7)).

- Since the reduced $\chi^{2}$ of the fit has been found to be significantly greater than unity, we conclude that either we have underestimated errors or, more likely, that the relatively large scatter around the FP (of about 0.15 in $\log R_{\mathrm{e}}$ ) is intrinsic. We found that the so-called GC streams are likely to be not responsible for the scatter around the FP.

- We have shown that in the standard FP coordinates the set of points representing our sample of GCs occupies a rather slim cylindrical region of parameter space, which suggests that the relevant scaling relation might be around a line, rather than a plane; this confirms a result noted previously by Bellazzini (1998). We think that this is the origin of the difficulties in fits by a plane noted earlier (e.g., see Djorgovski 1995; De Micheli 2003). In addition, we have derived eigenvalues and eigenvectors for the tensor of inertia of the distribution of data-points in the $S B_{\mathrm{e}}, \log R_{\mathrm{e}}, \log \sigma$ coordinates and found that such distribution is remarkably axisymmetric around a line, mainly along $S B_{\mathrm{e}}$.

- For our sample, we found no statistically significant correlation of FP residuals with metallicity, colour, concentration parameter, position in the Galaxy, ages, and relaxation times, but we found a potentially interesting correlation with the central slope of the surface brightness profile.

- By means of kernel density estimation techniques, we have reconstructed the probability density distribution of the effective virial coefficient $K_{V} /(M / L)$ of GCs, providing evidence for bimodality. We argue that this feature is not related to the standard disk/halo dichotomy, because our sample comprises only clusters with metallicity below about -0.8 , which are therefore likely to be part of the halo population.

- By means of non-parametric statistical tests, we studied the selection effects affecting our sample and the sample used by Djorgovski (1995) in his pioneering paper on the FP for the galactic GCs, demonstrating that some selection effects are present (in particular, our sample is biased in the direction of bright, metal poor clusters close to the Sun). No significant selection effects are present in the relaxation time referred to the effective radius, which is reassuring because it suggests that our sample is not dynamically different from the remaining population of galactic GCs.

\subsection{Discussion and conclusions}

The error bars on the FP coefficients are rather large. Therefore, on the basis of currently available data it is difficult to tell whether the FP relation of GCs is a continuation of that of earlytype galaxies. The limited luminosity range of galactic GCs may be an issue here; studies of extragalactic GC systems such as that of NGC 5128, which reach a higher upper luminosity limit (e.g., see Rejkuba et al. 2007; Martini \& Ho 2004), are consistent with the present study. In principle, the current FP fit does not exclude (at least at a marginal level) that a no-tilt relation is at the basis of the observed correlations. On the other hand, we found indications that the shape of the distribution of $K_{V} /(M / L)$ is bimodal; if such indications are confirmed, this would argue against the naive interpretation that the FP just reflects the virial constraint.

The hints of a bimodal distribution of the effective virial coefficient $K_{V} /(M / L)$ lead us to consider one specific physical ingredient that might play an important role in the statistical properties of the GC system, that is, the possible presence of intermediate mass black holes. Such a presence would also have a counterpart in the second unexpected feature noted in our statistical investigation, i.e., the trend of FP residuals with the central slope of surface brightness profiles.

As to the possibility that the Fundamental Plane should be replaced, for globular clusters, by a Fundamental Line relation, before proposing a physical interpretation we prefer to wait for confirmation from a wider sample or from studies of globular cluster systems in external galaxies. The possibility of answering to this issue soon is reasonable because, as discussed at the end of Sect. 3, the Fundamental Line relation appears to imply the existence of a pure photometric scaling law.

Acknowledgements. We wish to thank L. Ciotti, S. Degl'Innocenti, F. Ferraro, M. Lombardi, E. Noyola, G. Punzi, S. Trager, M. Trenti, and E. Vesperini for their comments and helpful suggestions. We also thank the Referee, for making a number of constructive remarks that have helped us improve the paper. The initial part of the work started in order to clarify some questions raised in the Thesis by De Micheli (2003). This research has made use of the SIMBAD database, operated at CDS, Strasbourg, France. This work was partially supported by the Italian MiUR.

\section{Appendix A: Discussion of the FP on two subsamples with uniform distance moduli}

For the sub-sample of 35 GCs with distance moduli from Ferraro et al. (1999) we find the following fit by the Fundamental Plane based on $S B_{\mathrm{e}}$ :

$S B_{\mathrm{e}}=(3.2 \pm 0.7) \log R_{\mathrm{e}}-(3.4 \pm 0.5) \log \sigma+(27.96 \pm 3.5)$.

In turn, fitting through $\log R_{\mathrm{e}}$ we obtain

$\log R_{\mathrm{e}}=(0.14 \pm 0.03) S B_{\mathrm{e}}+(0.3 \pm 0.1) \log \sigma-(5.04 \pm 0.6)$,

and, finally, through $\log \sigma$ :

$\log \sigma=-(0.18 \pm 0.02) S B_{\mathrm{e}}+(0.4 \pm 0.2) \log R_{\mathrm{e}}$

$+(5.15 \pm 0.6)$. 
Table A.1. Properties of the sample considered in this paper.

\begin{tabular}{|c|c|c|c|c|c|c|c|c|}
\hline ID & $\overline{\sigma^{a}}$ & $m^{b}$ & $\log r_{\mathrm{e}}^{c}$ & $|M-m|_{0}^{d}$ & $\bar{\delta} \delta|M-m|_{0}^{e}$ & $E(B-V)^{f}$ & $\mathrm{~d} \log \Sigma / \mathrm{d} \log r^{g}$ & Distance sample $^{h}$ \\
\hline NGC 104 & 9.72 & 4.00 & 2.22 & 13.27 & 0.08 & 0.04 & $0.00 \pm 0.04$ & R05 F99 \\
\hline NGC 288 & 2.61 & 8.45 & 2.16 & 14.67 & 0.30 & 0.03 & & F99 \\
\hline NGC 362 & 6.15 & 6.26 & 1.69 & 14.69 & 0.10 & 0.05 & & R05 F99 \\
\hline NGC 1851 & 10.21 & 7.32 & 1.46 & 15.44 & 0.09 & 0.02 & $-0.38 \pm 0.11$ & R05 F99 \\
\hline NGC 1904 & 4.59 & 7.70 & 1.93 & 15.67 & 0.08 & 0.01 & $-0.03 \pm 0.07$ & R05 F99 \\
\hline NGC 2419 & 2.66 & 10.46 & 1.17 & 19.88 & 0.29 & 0.03 & & F99 \\
\hline NGC 2808 & 14.04 & 6.42 & 1.77 & 14.96 & 0.13 & 0.23 & $-0.06 \pm 0.07$ & R05 F99 \\
\hline NGC 3201 & 4.30 & 7.21 & 2.30 & 13.58 & 0.13 & 0.21 & & R05 F99 \\
\hline NGC 4147 & 2.54 & 10.32 & 1.46 & 16.42 & 0.10 & 0.02 & & R05 F99 \\
\hline NGC 4590 & 2.39 & 8.32 & 1.96 & 15.16 & 0.07 & 0.04 & & R05 F99 \\
\hline NGC 5053 & 1.15 & 9.79 & 2.29 & 16.17 & 0.19 & 0.03 & & F99 \\
\hline NGC 5272 & 5.22 & 6.65 & 1.83 & 14.99 & 0.14 & 0.01 & $-0.05 \pm 0.10$ & F99 \\
\hline NGC 5286 & 8.00 & 7.41 & 1.90 & 15.25 & 0.29 & 0.24 & $-0.28 \pm 0.11$ & F99 \\
\hline NGC 5466 & 1.57 & 9.11 & 2.13 & 16.12 & 0.29 & 0.00 & & F99 \\
\hline NGC 5694 & 5.50 & 10.17 & 1.43 & 17.80 & 0.11 & 0.09 & $-0.19 \pm 0.11$ & R05 F99 \\
\hline NGC 5824 & 10.73 & 8.90 & 1.44 & 17.63 & 0.11 & 0.13 & $-0.36 \pm 0.16$ & R05 F99 \\
\hline NGC 5904 & 5.24 & 5.93 & 1.97 & 14.44 & 0.09 & 0.03 & $0.05 \pm 0.07$ & R05 F99 \\
\hline NGC 5946 & 3.70 & 9.74 & 1.62 & 15.41 & 0.29 & 0.55 & & R05 \\
\hline NGC 6093 & 13.32 & 7.47 & 1.56 & 15.09 & 0.15 & 0.18 & $-0.16 \pm 0.07$ & R05 F99 \\
\hline NGC 6121 & 3.59 & 5.67 & 2.34 & 11.62 & 0.30 & 0.36 & & F99 \\
\hline NGC 6171 & 3.67 & 8.29 & 2.16 & 14.07 & 0.17 & 0.33 & & R05 F99 \\
\hline NGC 6205 & 6.62 & 6.00 & 1.96 & 14.45 & 0.08 & 0.02 & $-0.10 \pm 0.15$ & R05 F99 \\
\hline NGC 6218 & 3.92 & 7.27 & 2.02 & 13.66 & 0.23 & 0.18 & & R05 F99 \\
\hline NGC 6254 & 5.48 & 6.70 & 2.08 & 13.35 & 0.29 & 0.28 & $0.05 \pm 0.07$ & F99 \\
\hline NGC 6256 & 6.50 & 10.91 & 1.89 & 14.97 & 0.37 & 0.84 & & R05 \\
\hline NGC 6266 & 14.23 & 6.50 & 1.81 & 14.31 & 0.21 & 0.47 & $-0.13 \pm 0.08$ & R05 F99 \\
\hline NGC 6284 & 6.20 & 8.82 & 1.70 & 16.08 & 0.21 & 0.28 & $-0.55 \pm 0.14$ & R05 \\
\hline NGC 6293 & 7.60 & 8.28 & 1.76 & 14.88 & 0.24 & 0.39 & $-0.67 \pm 0.08$ & R05 \\
\hline NGC 6325 & 5.80 & 10.30 & 1.88 & 14.82 & 0.39 & 0.89 & & R05 \\
\hline NGC 6341 & 5.00 & 6.53 & 1.69 & 14.74 & 0.29 & 0.02 & $-0.01 \pm 0.04$ & F99 \\
\hline NGC 6342 & 4.60 & 9.80 & 1.68 & 15.00 & 0.26 & 0.44 & & R05 \\
\hline NGC 6366 & 0.93 & 9.27 & 2.26 & 12.88 & 0.30 & 0.69 & & F99 \\
\hline NGC 6362 & 2.76 & 7.71 & 2.24 & 14.45 & 0.16 & 0.09 & & R05 \\
\hline NGC 6388 & 18.90 & 6.95 & 1.54 & 15.38 & 0.24 & 0.38 & $-0.13 \pm 0.07$ & R05 \\
\hline NGC 6397 & 3.48 & 6.06 & 2.28 & 11.89 & 0.29 & 0.18 & $-0.37 \pm 0.11$ & F99 \\
\hline NGC 6441 & 17.98 & 7.35 & 1.50 & 15.82 & 0.26 & 0.45 & $-0.02 \pm 0.12$ & R05 \\
\hline NGC 6522 & 6.70 & 8.34 & 1.71 & 14.77 & 0.28 & 0.50 & & R05 \\
\hline NGC 6535 & 2.15 & 10.53 & 1.66 & 14.29 & 0.45 & 0.32 & $-0.50 \pm 0.18$ & F99 \\
\hline NGC 6624 & 5.54 & 8.00 & 1.66 & 14.45 & 0.21 & 0.27 & $-0.32 \pm 0.16$ & R05 \\
\hline NGC 6681 & 8.21 & 8.12 & 1.68 & 14.99 & 0.10 & 0.07 & $-0.82 \pm 0.09$ & R05 F99 \\
\hline NGC 6712 & 4.03 & 8.03 & 2.03 & 14.15 & 0.15 & 0.46 & $0.02 \pm 0.05$ & R05 F99 \\
\hline NGC 6752 & 4.50 & 5.75 & 2.06 & 13.13 & 0.45 & 0.04 & $-0.03 \pm 0.15$ & F99 \\
\hline NGC 6809 & 4.12 & 6.96 & 2.21 & 13.78 & 0.29 & 0.07 & & F99 \\
\hline NGC 6864 & 10.30 & 8.51 & 1.46 & 16.63 & 0.18 & 0.16 & & R05 \\
\hline NGC 6934 & 4.91 & 8.86 & 1.62 & 16.03 & 0.11 & 0.11 & & R05 F99 \\
\hline NGC 7078 & 12.98 & 6.42 & 1.78 & 15.19 & 0.11 & 0.09 & $-0.66 \pm 0.11$ & R05 F99 \\
\hline NGC 7089 & 7.39 & 6.46 & 1.85 & 15.35 & 0.15 & 0.05 & $0.05 \pm 0.11$ & R05 \\
\hline NGC 7099 & 5.13 & 7.48 & 1.93 & 14.72 & 0.08 & 0.03 & $-0.57 \pm 0.11$ & R05 F99 \\
\hline
\end{tabular}

${ }^{a} \mathrm{~km} \mathrm{~s}^{-1}$, Pryor \& Meylan (1993); ${ }^{b} V$-band mag, this paper; ${ }^{c}$ arcsec, this paper; ${ }^{d}$ mag, Ferraro et al. (1999) and Recio-Blanco et al. (2005); ${ }^{e}$ Ferraro et al. (1999); Recio-Blanco et al. (2005) and this paper (see text); ${ }^{f}$ mag, Recio-Blanco et al. (2005) and Harris (1996); ${ }^{g}$ dimensionless, Noyola \& Gebhardt (2006); ${ }^{h}$ F99 indicates that the GC is listed by Ferraro et al. (1999), R05 that it is listed by Recio-Blanco et al. (2005).

For the sub-sample of $34 \mathrm{GCs}$ with distance moduli from Recio-Blanco et al. (2005) we find the following fit by the Fundamental Plane based on $S B_{\mathrm{e}}$ :

$$
\begin{aligned}
S B_{\mathrm{e}}= & (2.66 \pm 0.75) \log R_{\mathrm{e}}-(4.02 \pm 0.47) \log \sigma \\
& +(31.70 \pm 3.9) .
\end{aligned}
$$

Fitting through $\log R_{\mathrm{e}}$ we obtain

$$
\begin{aligned}
\log R_{\mathrm{e}}= & (0.12 \pm 0.03) S B_{\mathrm{e}}+(0.26 \pm 0.18) \log \sigma \\
& -(5.19 \pm 0.65),
\end{aligned}
$$

and, finally, through $\log \sigma$ :

$$
\begin{aligned}
\log \sigma= & -(0.17 \pm 0.02) S B_{\mathrm{e}}+(0.36 \pm 0.14) \log R_{\mathrm{e}} \\
& +(5.16 \pm 0.52) .
\end{aligned}
$$

These results are compatible with those obtained using the global sample of 48 GCs considered in the main text of the paper. Since Recio-Blanco et al. (2005) and Ferraro et al. (1999) use a different definition of the ZAHB level, these results show that our conclusions (and in particular the FP coefficients) are relatively independent of the details of ZAHB fitting. 
We have also checked that the general results listed in Sect. 6.1 hold for the two subsamples if studied separately.

\section{References}

Barmby, P., McLaughlin, D. E., Harris, W. E., Harris, G. L. H., \& Forbes, D. A. 2007, AJ, 133, 2764

Bellazzini, M. 1998, New Astron., 3, 219

Bender, R., Burstein, D., \& Faber, S. M. 1992, ApJ, 399, 462

Bertin, G., Ciotti, L., \& Del Principe, M. 2002, A\&A, 386, 149

Bonatto, C., \& Bica, E. 2005, A\&A, 437, 483

Burstein, D., Bender, R., Faber, S., \& Nolthenius, R. 1997, AJ, 114, 1365

Byun, Y.-I., Grillmair, C. J., Faber, S. M., et al. 1996, AJ, 111, 1889

Capaccioli, M., Caon, N., \& D'Onofrio, M. 1992, MNRAS, 259, 323

Carney, B. W. 1996, PASP, 108, 900

Carretta, E., \& Gratton, R. G. 1997, A\&AS, 121, 95

Cipollina, M., \& Bertin, G. 1994, A\&A, 288, 43

De Angeli, F., Piotto, G., Cassisi, S., et al. 2005, AJ, 130, 116

De Micheli, F. 2003, Tesi di Laurea, Università degli Studi di Milano

Diehl, S., \& Statler, T. S. 2005, ApJ, 633, L21

Djorgovski, S. 1995, ApJ, 438, L29

Djorgovski, S., \& Davis, M. 1987, ApJ, 313, 59

Djorgovski, S., \& Meylan, G. 1994, AJ, 108, 1292

Dressler, A., Lynden-Bell, D., Burstein, D., et al. 1987, ApJ, 313, 42

Faber, S. M., Tremaine, S., Ajhar, E. A., et al. 1997, AJ, 114, 1771

Ferrarese, L., \& Merritt, D. 2000, ApJ, 539, L9

Ferraro, F. R., Messineo, M., Fusi Pecci, F., et al. 1999, AJ, 118, 1738

Fish, R. A. 1964, ApJ, 139, 284

Gao, S., Jiang, B.-W., \& Zhao, Y.-H. 2007, Chinese J. Astron. Astrophys., 7, 111

Gebhardt, K., Bender, R., Bower, G., et al. 2000, ApJ, 539, L13

Hamabe, M., \& Kormendy, J. 1987, in Structure and Dynamics of Elliptical Galaxies, ed. P. T. de Zeeuw, IAU Symp., 127, 379
Hamilton, T. S., Casertano, S., \& Turnshek, D. A. 2006, New Astron. Rev., 50, 758

Harris, W. E. 1996, AJ, 112, 1487

Holtzman, J. A., Burrows, C. J., Casertano, S., et al. 1995, PASP, 107, 1065

Hopkins, P. F., Hernquist, L., Cox, T. J., Robertson, B., \& Krause, E. 2007, ApJ, 669,67

Jørgensen, I., Franx, M., \& Kjaergaard, P. 1996, MNRAS, 280, 167

King, I. R. 1966, AJ, 71, 64

Kormendy, J. 1977, ApJ, 218, 333

Lanzoni, B., Ciotti, L., Cappi, A., Tormen, G., \& Zamorani, G. 2004, ApJ, 600, 640

Lynden-Bell, D., \& Lynden-Bell, R. M. 1995, MNRAS, 275, 429

Martini, P., \& Ho, L. C. 2004, ApJ, 610, 233

McLaughlin, D. E. 2000, ApJ, 539, 618

McLaughlin, D. E., \& van der Marel, R. P. 2005, ApJS, 161, 304

Noyola, E., \& Gebhardt, K. 2006, AJ, 132, 447

Noyola, E., Gebhardt, K., \& Bergmann, M. 2008, ApJ, 676, 1008

Piotto, G., King, I. R., Djorgovski, S. G., et al. 2002, A\&A, 391, 945

Pryor, C., \& Meylan, G. 1993, in Structure and Dynamics of Globular Clusters, ed. S. G. Djorgovski \& G. Meylan, ASP Conf. Ser., 50, 357

Recio-Blanco, A., Piotto, G., de Angeli, F., et al. 2005, A\&A, 432, 851

Rejkuba, M., Dubath, P., Minniti, D., \& Meylan, G. 2007, A\&A, 469, 147

Salaris, M., \& Cassisi, S. 1996, A\&A, 305, 858

Salaris, M., Chieffi, A., \& Straniero, O. 1993, ApJ, 414, 580

Trager, S. C., King, I. R., \& Djorgovski, S. 1995, AJ, 109, 218

Treu, T., Stiavelli, M., Casertano, S., Møller, P., \& Bertin, G. 1999, MNRAS, 308,1037

Wasserman, L. 2006, All of Nonparametric Statistics (Springer Texts in Statistics) (Springer Science+Business Media Inc.)

Zaritsky, D., Gonzalez, A. H., \& Zabludoff, A. I. 2006, ApJ, 638, 725

Zoccali, M., Ortolani, S., Renzini, A., et al. 1999, in Harmonizing Cosmic Distance Scales in a Post-HIPPARCOS Era, ed. D. Egret, \& A. Heck, ASP Conf. Ser., 167, 336 